\title{
Influenza Vaccination of Pregnant Women and Protection of Their Infants
}

\author{
Shabir A. Madhi, M.D., Ph.D., Clare L. Cutland, M.D., Locadiah Kuwanda, M.Sc., \\ Adriana Weinberg, M.D., Andrea Hugo, M.D., Stephanie Jones, M.D., \\ Peter V. Adrian, Ph.D., Nadia van Niekerk, B.Tech., Florette Treurnicht, Ph.D., \\ Justin R. Ortiz, M.D., Marietjie Venter, Ph.D., Avy Violari, M.D., \\ Kathleen M. Neuzil, M.D., Eric A.F. Simões, M.D., Keith P. Klugman, M.D., Ph.D., \\ and Marta C. Nunes, Ph.D., for the Maternal Flu Trial (Matflu) Team*
}

ABSTRACT

The authors' affiliations are listed in the Appendix. Address reprint requests to Dr. Madhi or Dr. Nunes at the Respiratory and Meningeal Pathogens Research Unit, Chris Hani Rd., Chris Hani-Baragwanath Hospital, New Nurses Residencelst Fl. West Wing, Bertsham, Gauteng 2013, South Africa, or at shabirm@nicd .ac.za or nunesm@rmpru.co.za.

*A complete list of investigators in the Matflu Trial is available in the Supplementary Appendix, available at NEJM.org.

Drs. Madhi and Nunes contributed equally to this article.

N EnglJ Med 2014;371:918-31. DOI: 10.1056/NEJMoa1401480 Copyright (C) 2014 Massachusetts Medical Society.

\section{BACKGROUND}

There are limited data on the efficacy of vaccination against confirmed influenza in pregnant women with and those without human immunodeficiency virus (HIV) infection and protection of their infants.

\section{METHODS}

We conducted two double-blind, randomized, placebo-controlled trials of trivalent inactivated influenza vaccine (IIV3) in South Africa during 2011 in pregnant women infected with HIV and during 2011 and 2012 in pregnant women who were not infected. The immunogenicity, safety, and efficacy of IIV3 in pregnant women and their infants were evaluated until 24 weeks after birth. Immune responses were measured with a hemagglutination inhibition (HAI) assay, and influenza was diagnosed by means of reverse-transcriptase-polymerase-chain-reaction (RT-PCR) assays of respiratory samples.

RESULTS

The study cohorts included 2116 pregnant women who were not infected with HIV and 194 pregnant women who were infected with HIV. At 1 month after vaccination, seroconversion rates and the proportion of participants with HAI titers of 1:40 or more were higher among IIV3 recipients than among placebo recipients in both cohorts. Newborns of IIV3 recipients also had higher HAI titers than newborns of placebo recipients. The attack rate for RT-PCR-confirmed influenza among both HIV-uninfected placebo recipients and their infants was 3.6\%. The attack rates among HIV-uninfected IIV3 recipients and their infants were $1.8 \%$ and $1.9 \%$, respectively, and the respective vaccine-efficacy rates were $50.4 \%$ ( $95 \%$ confidence interval [CI], 14.5 to 71.2 ) and $48.8 \%$ (95\% CI, 11.6 to 70.4). Among HIV-infected women, the attack rate for placebo recipients was $17.0 \%$ and the rate for IIV3 recipients was $7.0 \%$; the vaccine-efficacy rate for these IIV3 recipients was $57.7 \%$ (95\% CI, 0.2 to 82.1 ).

\section{CONCLUSIONS}

Influenza vaccine was immunogenic in HIV-uninfected and HIV-infected pregnant women and provided partial protection against confirmed influenza in both groups of women and in infants who were not exposed to HIV. (Funded by the Bill and Melinda Gates Foundation and others; ClinicalTrials.gov numbers, NCT01306669 and NCT01306682.) 
P REGNANT WOMEN ARE DESIGNATED AS A priority group for seasonal influenza vaccination by the World Health Organization $(\mathrm{WHO})^{1}$ because of their heightened susceptibility to severe influenza from the second trimester to the early postpartum period. ${ }^{2,3}$ Since pregnancy is associated with immunomodulation, including the attenuation of cell-mediated immune responses, ${ }^{4}$ the efficacy of inactivated influenza vaccine (IIV) in pregnant women may differ from its efficacy in healthy nonpregnant women and in men. ${ }^{5}$ This difference in vaccine efficacy could be further accentuated in pregnant women infected with the human immunodeficiency virus (HIV), who are at heightened risk for severe influenza illness ${ }^{6-8}$ because of HIV-related immunosuppression. ${ }^{9-14}$

Reduced attack rates for all-cause febrile respiratory illness among women vaccinated against influenza during pregnancy were observed during the 1957 Asian influenza pandemic in the United States and in a later randomized, controlled trial in Bangladesh. ${ }^{15,16}$ A recent case-control study in the United States also reported that pregnant women with influenza confirmed by means of reverse-transcriptase-polymerase-chainreaction (RT-PCR) assay (hereafter referred to as confirmed influenza) were 44 to $53 \%$ less likely to have been vaccinated with trivalent IIV (IIV3) than controls who did not have influenza. ${ }^{17}$ To our knowledge, no published randomized, controlled trial has assessed the efficacy of IIV in preventing confirmed influenza in pregnant women with HIV infection and those without HIV infection. ${ }^{18,19}$

The vaccination of pregnant women may also confer partial protection against confirmed influenza in their infants, as reported in the Bangladeshi trial (in which infants whose mothers had been vaccinated with IIV3 were $63 \%$ less likely to have influenza [confirmed by means of enzyme-linked immunosorbent assay]). ${ }^{16}$ However, observational studies have had conflicting results with regard to the efficacy of IIV3 vaccination during pregnancy in protecting infants against all-cause respiratory illness. ${ }^{20-23}$ The protection of infants 6 months of age or younger against influenza is a public health priority. These infants are at high risk for influenza-associated hospitalization, and their immune responses to IIV vaccination are poor. ${ }^{24-26}$ We conducted two studies, one involving pregnant women without
HIV infection and the other involving pregnant women with HIV infection, to evaluate the safety, immunogenicity, and efficacy of IIV3 in these women and in their infants until 24 weeks post partum.

\section{METHODS}

STUDY DESIGN, OBJECTIVES, AND OVERSIGHT

The two studies were randomized, double-blind, placebo-controlled trials conducted in Soweto, South Africa, where antenatal HIV testing is routine. The enrollment of HIV-uninfected pregnant women was initiated at four antenatal clinics before the onset of the 2011 influenza season (March 3 through August 4) and the 2012 season (March 6 through July 2). Members of the HIVinfected cohort were enrolled at the same facilities (March 3 through June 2, 2011). Eligibility criteria for both cohorts included an age of 18 to 38 years, an estimated gestation of 20 to 36 weeks, and the ability to understand and comply with planned study procedures. (The methods used to determine gestational age and the exclusion criteria are provided in the Supplementary Appendix, which is available with the full text of this article, along with the original and final protocols, at NEJM.org.) All HIV-infected mother-infant dyads in 2011 and a subset of HIV-uninfected dyads were included in a nested immunogenicity study that was performed during each year of the trial. The primary objectives for the cohort of women without HIV infection were to evaluate the efficacy of IIV3 vaccination during pregnancy in protecting their infants against confirmed influenza through 24 weeks of age and to compare seroconversion rates between IIV3 recipients and placebo recipients 1 month after vaccination. Secondary objectives included measuring vaccine efficacy against confirmed influenza in all women until 24 weeks post partum. In the HIV-infected cohort, an additional primary objective was to evaluate the immunogenicity of IIV3 in the women. Secondary objectives for this cohort included measuring vaccine efficacy against confirmed influenza in the women and their infants until 24 weeks post partum. Additional secondary objectives for both cohorts are listed in the Supplementary Appendix.

The studies were approved by the Human Research Ethics Committee of the University of the Witwatersrand and were conducted in accor- 
dance with Good Clinical Practice guidelines. Written informed consent was obtained from all participants. All the authors vouch for the fidelity of this report to the protocols and the completeness of the data and analyses. The funders did not participate in the conduct of the study, data collection, analyses of the data, or the writing of the manuscript.

\section{RANDOMIZATION AND STUDY TREATMENT}

Participants were randomly assigned in a 1:1 ratio to receive IIV3 or placebo. Randomization was performed by the study statistician with the use of computer-generated assignments. With the exception of the statistician and the pharmacist, study personnel were unaware of the group assignments, as were the study participants.

Influenza vaccine (Vaxigrip, lot number G05831 in 2011 and H7221-2 in 2012; Sanofi Pasteur) was purchased by the study team. The vaccine contained $15 \mu \mathrm{g}$ each of A/California/7/2009 (A/[H1N1]pdm09), A/Victoria/210/2009 (A/H3N2), and a $\mathrm{B} /$ Brisbane/60/2008-like virus (B/Victoria), as recommended by WHO for the Southern Hemisphere in 2011 and 2012..$^{27,28}$ The study pharmacist used a 2-ml syringe to draw $0.5 \mathrm{ml}$ of vaccine for the women receiving IIV3 and $0.5 \mathrm{ml}$ of sterile $0.9 \%$ normal saline solution for the women receiving placebo. The two preparations were macroscopically indistinguishable. The vaccines were administered into the deltoid muscle by study staff.

\section{SEROLOGIC EFFICACY AND IMMUNOGENICITY}

In 2011 and 2012, all mothers and infants in the HIV-infected cohort and a nested group of 180 HIV-uninfected mother-infant dyads were enrolled in an intensive safety and immunogenicity study. The timing of blood-sample collection and processing, the method used for antibody testing (hemagglutination inhibition [HAI]), and the standard criteria used to qualify HAI results as seroconversion and as seroprotective or seronegative titers are provided in the Supplementary Appendix.

\section{VACCINE EFFICACY}

Active surveillance for influenza-like illness (as defined in the protocols) was conducted through weekly contact with participants (see the Supplementary Appendix for details). Women and infants identified as having influenza-like illness, and those unexpectedly presenting with or hospitalized for any respiratory illness, were tested for influ- enza virus by means of an RT-PCR assay. The laboratory methods used for sample collection, influenza-virus identification, and genotyping are detailed in the Supplementary Appendix.

\section{SAFETY}

Women enrolled in the nested immunogenicity subsets were provided with diary cards on which to document possible local and systemic reactions to vaccination for 1 week. Digital thermometers were provided to measure oral temperature in women and axillary temperature in infants after vaccination and during illness. Serious adverse events were recorded and graded throughout the study period with the use of an established system. ${ }^{29}$

\section{STATISTICAL ANALYSIS}

In the HIV-uninfected cohort, the sample size was based on the primary outcome of vaccine efficacy in the infants. The sample size for the HIV-uninfected cohort was outcome-driven. We aimed at identifying at least 27 cases of confirmed illness caused by influenza virus in infants up to 24 weeks of age in order to detect a 70\% reduction in confirmed influenza among the infants, with $80 \%$ power. The sample size required for the HIVinfected cohort was 180 participants, which provided $90 \%$ power to detect a difference of at least $67 \%$ in rates of seroconversion to individual vaccine strains between IIV3 recipients and placebo recipients.

The immunogenicity analyses included comparisons of geometric mean titers between study groups and of the increase in titers from baseline to 1 month after vaccination. We performed a two-sided, two-sample t-test and calculated the corresponding $95 \%$ confidence intervals for the titers, using logarithmic transformation for all values. Post-vaccination analyses of immune response were adjusted for baseline HAI titers and between-group differences in baseline characteristics. The proportions of participants in each group who underwent seroconversion and the proportions of participants who had local or systemic reactions were compared by means of chisquare or Fischer's exact tests. Vaccine efficacy was calculated with the use of the formula $\left(1-\mathrm{I}_{\mathrm{L}}\right) /$ $I_{p}$, where $I_{L}$ is the case incidence rate in the vaccinated group and $I_{p}$ is the case incidence rate in the placebo group; $95 \%$ confidence intervals were calculated and between-group differences were 
tested. Estimates of vaccine efficacy were adjusted for differences in maternal age and status with respect to antiretroviral treatment at enrollment, which were prevalent in the HIV-infected cohort. For vaccine efficacy end points, data were censored after the first episode of a specific clinical outcome. Between-group differences in the time to a first episode of confirmed influenza were compared in survival analyses by means of the log-rank test.

The intention-to-treat analyses included maternal outcomes from receipt of vaccine or placebo to 175 days after birth and infant outcomes from birth to 175 days of age. The per-protocol analyses included maternal outcomes that occurred 14 or more days after receipt of vaccine or placebo; perprotocol analyses of outcomes for infants were limited to those born at least 28 days after their mother's vaccination, those whose gestational age at birth was at least 37 weeks, or those who had a birth weight of at least $2500 \mathrm{~g}$. The per-protocol immunogenicity analysis was limited to women from whom a blood sample was obtained 28 to 35 days after vaccination and to infants from whom a blood sample was obtained within 7 days after birth and who had a gestational age of at least 37 weeks at birth or a birth weight of at least $2500 \mathrm{~g}$. An exploratory analysis was performed with the use of extended windows for obtaining blood samples after vaccination ( 28 to 42 days) and after delivery (up to 14 days).

Study data were collected and managed with the use of Research Electronic Data Capture (REDCap), version 5.9.13. ${ }^{30}$ All statistical analyses were conducted with the use of Stata software, version 12.1. All $\mathrm{P}$ values were two-sided, and a value of 0.05 or less was considered to indicate statistical significance.

\section{RESULTS}

BASELINE CHARACTERISTICS OF THE STUDY PARTICIPANTS

We enrolled 2116 black African pregnant women who were not infected with HIV; 1062 were randomly assigned to the group vaccinated with IIV3 and 1054 to the group that received placebo. There were 1026 live infants born to IIV3 recipients and 1023 live infants born to placebo recipients (Fig. S1 in the Supplementary Appendix). The mean maternal age at enrollment was 26.2 years, and the mean gestational age was 26.8 weeks (Table 1 ).
We enrolled 194 pregnant women who were infected with HIV; 100 were randomly assigned to the group vaccinated with IIV3 and 94 to the placebo group (Fig. S2 in the Supplementary Appendix). The mean maternal age at enrollment was 28.2 years, and the mean gestational age was 27.3 weeks (Table 2). The baseline median CD4+ T-cell count in the HIV-infected women was 393.5 cells per cubic millimeter; $12.6 \%$ of the women (24 of 190) had counts of less than 200 cells per cubic millimeter. The median level of plasma HIV-1 RNA was 1067 copies per milliliter; 23.0\% of the women (43 of 187) had undetectable levels of HIV-1 RNA. There were 100 live births in the group vaccinated with IIV3 and 88 live births in the placebo group among the 183 HIV-infected women who remained in the study until delivery.

\section{IMMUNOGENICITY OF THE VACCINE HIV-Uninfected Cohort}

Of the 376 participants in the immunogenicity subset, 142 IIV3 recipients and 148 placebo recipients were included in the per-protocol analysis of immunogenicity (Fig. S3 and Table S1 in the Supplementary Appendix). Post-vaccination geometric mean titers increased from baseline by a factor of 6 to 10 in IIV3 recipients and were significantly higher for all three vaccine strains, as compared with titers in placebo recipients (Table 3). Seroconversion rates for strains A(H1N1) pdm09, A(H3N2), and B(Victoria) among IIV3 recipients were $72.5 \%, 64.8 \%$, and $92.3 \%$, respectively; rates among placebo recipients were $8.1 \%$, $2.7 \%$, and $2.0 \%$, respectively ( $\mathrm{P}<0.001$ for all comparisons). A greater proportion of IIV3 recipients had seroprotective HAI titers after vaccination, as compared with placebo recipients (Table 3).

Newborns of IIV3 recipients had higher HAI geometric mean titers for all vaccine strains than did newborns of placebo recipients and were also more likely to have an HAI titer of 1:40 or higher for $\mathrm{A} /(\mathrm{H} 1 \mathrm{~N} 1) \mathrm{pdm} 09$ (81.1\% vs. $34.0 \%), \mathrm{A}(\mathrm{H} 3 \mathrm{~N} 2)$ (60.0\% vs. $17.5 \%)$, and $\mathrm{B}$ (Victoria) $(82.1 \%$ vs. 43.7\%) ( $\mathrm{P}<0.001$ for all comparisons) (Table 3). The ratios of HAI titers in newborns to maternal titers were 0.7 to 1.0 for the three vaccine strains and were similar between study groups, with the exception of higher ratios for B/Victoria in the placebo group (Table 3). Similar findings were observed in the immunogenicity analyses when the window for obtaining blood samples was extended (Tables S2 and S3 in the Supplementary Appendix). 
Table 1. Baseline Characteristics of HIV-Uninfected Pregnant Women, Fetal Outcomes, and Newborn Characteristics.*

\begin{tabular}{|c|c|c|c|}
\hline Characteristic or Outcome & Overall & IIV3 & Placebo \\
\hline \multicolumn{4}{|l|}{ Women } \\
\hline Total no. of women & 2116 & 1062 & 1054 \\
\hline Mean age $-y r$ & $26.2 \pm 5.3$ & $26.4 \pm 5.3$ & $25.9 \pm 5.3$ \\
\hline \multicolumn{4}{|l|}{ Body-mass index' } \\
\hline Median & 27.6 & 28.0 & 27.4 \\
\hline Interquartile range & $24.3-31.9$ & $24.6-32.1$ & $24.1-31.6$ \\
\hline Mean gestational age at enrollment $-w k$ & $26.8 \pm 4.4$ & $26.7 \pm 4.4$ & $26.9 \pm 4.4$ \\
\hline \multicolumn{4}{|l|}{ Gravidity } \\
\hline Median & 2.0 & 2.0 & 2.0 \\
\hline Interquartile range & $0.0-3.0$ & $0.0-3.0$ & $0.0-3.0$ \\
\hline \multicolumn{4}{|l|}{ Parity } \\
\hline Median & 1.0 & 1.0 & 1.0 \\
\hline Interquartile range & $0.0-1.0$ & $0.0-1.0$ & $0.0-1.0$ \\
\hline \multicolumn{4}{|l|}{ Fetuses and newborns } \\
\hline Total no. of known outcomes & 2081 & 1044 & 1037 \\
\hline Miscarriage, gestational age $<28 w k-$ no. $(\%) \$$ & $8(0.4)$ & $3(0.3) \int$ & $5(0.5)$ \\
\hline Stillbirth, gestational age $\geq 28 \mathrm{wk}-$ no. $(\%) \ddagger$ & $24(1.2)$ & $15(1.4)$ & $9(0.9)$ \\
\hline Live birth — no. (\%) & $2049(98.5)$ & $1026(98.3)$ & $1023(98.7)$ \\
\hline Twin birth — no. (\%) & $110(5.3)$ & $60(5.7)$ & $50(4.8)$ \\
\hline \multicolumn{4}{|l|}{ Type of delivery — no. (\%) } \\
\hline Normal vaginal delivery & $1393(66.9)$ & $700(67.0)$ & $693(66.8)$ \\
\hline Cesarian section & $656(31.5)$ & $326(31.2)$ & $330(31.8)$ \\
\hline Preterm birth, gestational age $<37$ wk - no. (\%) & $204(10.0)$ & $108(10.5)$ & $96(9.4)$ \\
\hline
\end{tabular}

Overall birth weight $-\mathrm{kg}\|\| * * *$

Median

\section{1}

Range

Birth weight of infants born during influenza season $-\mathrm{kg} q * * \cdots+\dagger$

$$
0.5-4.8
$$

3.1
$0.4-4.8$
$255(12.5)$
$22(1.1)$
$142(6.9)$

3.1

$0.5-4.6$

$0.7-4.8$

Median

Range

Birth weight of $<2500 \mathrm{~g}$ - no. (\%) $9 \| * * * *$

Death after delivery - no. (\%) $9+$

Admission to neonatal nursery — no. (\%)

* Plus-minus values are means \pm SD. HIV denotes human immunodeficiency virus, and IIV 3 trivalent inactivated influ enza vaccine.

$\dagger \quad$ The calculations for body-mass index (the weight in kilograms divided by the square of the height in meters) were based on 796 women in the IIV3 group and 789 women in the placebo group.

$\mp$ Details on miscarriages and stillbirths are provided in Table $S 9$ in the Supplementary Appendix.

$\int$ This number includes one medically assisted termination of pregnancy.

9 Numbers and percentages are based on live births.

$\|$ Numbers are based on 1024 observations in the IIV3 group and 1021 observations in the placebo group.

$*$ No significant difference in birth weight between the study groups was observed, even when weight was stratified according to sex.

†† Numbers are based on 857 observations in the IIV3 group and 874 observations in the placebo group.

+ Further details on newborn deaths are provided in Table S10 in the Supplementary Appendix. 


\section{HIV-Infected Cohort}

The demographic characteristics of HIV-infected women included in the per-protocol analysis of immunogenicity (Fig. S2 in the Supplementary Appendix) are shown in Table S4 in the Supplementary Appendix. The receipt of IIV3 resulted in increases in geometric mean titers of antibodies to $\mathrm{A} /(\mathrm{H} 1 \mathrm{~N} 1) \mathrm{pdm} 09, \mathrm{~A} / \mathrm{H} 3 \mathrm{~N} 2$, and $\mathrm{B} /$ Victoria by factors of 2.9, 2.4, and 3.2, respectively, and seroconversion rates of $42.9 \%, 35.7 \%$, and $40.0 \%$, respectively. Furthermore, as compared with placebo recipients, a higher proportion of IIV3 recipients had seroprotective HAI titers after vaccination (Table S5 in the Supplementary Appendix).

The newborns of IIV3 recipients had higher HAI geometric mean titers than did the newborns of placebo recipients. The ratio of geometric mean HAI titers ranged between 0.7 and 1.4; ratios were higher in the placebo group for $A /(H 1 N 1)$ pdm09 $(\mathrm{P}=0.05)$ and $\mathrm{A} / \mathrm{H} 3 \mathrm{~N} 2(\mathrm{P}=0.01)$. In the IIV3 group, the proportion of newborns with an HAI titer of $1: 40$ or higher was $60.7 \%$ for $A /(\mathrm{H} 1 \mathrm{~N} 1)$ pdm09, $42.9 \%$ for $\mathrm{A} / \mathrm{H} 3 \mathrm{~N} 2$, and $78.6 \%$ for $\mathrm{B} /$ Victoria; titers for all three strains were higher than those in the placebo group (Table S5 in the Supplementary Appendix). Similar findings were observed in the immunogenicity analyses when extended windows for obtaining blood samples were used (Tables S6 and S7 in Supplementary Appendix).

\section{VACCINE EFFICACY}

\section{HIV-Uninfected Cohort}

The attack rates for confirmed influenza among placebo recipients and IIV3 recipients were 3.6\% and $1.8 \%$, respectively, indicating a vaccine efficacy of 50.4\% (95\% confidence interval [CI], 14.5 to 71.2) (Table 4 and Fig. 1). The vaccine efficacy was similar when determined according to the vaccine strain $(46.1 \%$; $95 \%$ CI, 6.4 to 69.0$)$. For the 2 years of the study, the predominant strain of influenza virus circulating among placebo recipients was A/H3N2 (57.9\%), for which an exploratory analysis identified a vaccine efficacy of $57.5 \%$ (95\% CI, 7.6 to 80.4).

The attack rate was lower among infants whose mothers were IIV3 recipients (1.9\%) than among those whose mothers were placebo recipients (3.6\%), indicating a vaccine efficacy of $48.8 \%$ (95\% CI, 11.6 to 70.4) (Table 4 and Fig. 1). The predominant strains causing illness in the 38 in- fants of placebo recipients were A/H3N2 (14 infants [37\%]), B/Victoria (11 [29\%]), and B/Yamagata (12 [32\%]). Among infants with confirmed influenza, the numbers infected with the influenza virus strain carried by their mothers were as follows: 1 of 19 infants in the IIV3 group (5.3\%) and 11 of 37 in the placebo group $(29.7 \%, P=0.04)$. The vaccine efficacy estimates in the per-protocol analysis were similar to the estimates in the intention-to-treat analysis for the women and their infants (Table S8 in the Supplementary Appendix).

\section{HIV-Infected Cohort}

In the cohort of HIV-infected mothers, the attack rate was lower among IIV3 recipients than among placebo recipients ( $7.0 \%$ vs. $17.0 \%$ ), indicating an adjusted vaccine efficacy of $57.7 \%$ ( $95 \% \mathrm{CI}$, 0.2 to 82.1) (Table 4 and Fig. 1). The attack rates were $5.0 \%$ and $6.8 \%$ among infants of IIV3 recipients and infants of placebo recipients, respectively (vaccine efficacy, 26.7\%; $\mathrm{P}=0.60$ ) (Table 4 and Fig. 1). In 6 of 11 infants of HIV-infected mothers with confirmed influenza (55\%), including 2 infants whose mothers received IIV3, the mothers were infected with the same strain as their infants. Estimates of vaccine efficacy among HIV-infected women and their infants were similar in the per-protocol and intention-to-treat analyses (Table S8 in the Supplementary Appendix).

\section{SAFETY}

The details of solicited local and systemic reactions in the HIV-uninfected cohort and the HIVinfected cohort are shown in Tables S9 and S10 in the Supplementary Appendix. Injection-site reactions (mainly mild to moderate) were more frequent among IIV3 recipients than among placebo recipients in both cohorts, but there were no other significant differences in solicited reactions between the two study groups in either cohort. Data on serious adverse events in both cohorts, including infant and maternal deaths and hospitalizations, are shown in Tables S11 to S23 in the Supplementary Appendix. There were no significant between-group differences with regard to rates of miscarriage, stillbirth, or premature birth or birth weight in the HIV-uninfected cohort (Table 1, and Table S11 in the Supplementary Appendix) and in the HIV-infected cohort (Table 2). The findings were similar in an analy- 


\begin{tabular}{|c|c|c|c|}
\hline Characteristic or Outcome & Overall & IIV3 & Placebo \\
\hline \multicolumn{4}{|l|}{ Women } \\
\hline Total no. of women & 194 & 100 & 94 \\
\hline Mean age $-\mathrm{yr}$ & $28.2 \pm 5.1$ & $27.2 \pm 4.9 \dagger$ & $29.2 \pm 5.1 \dagger$ \\
\hline Mean body-mass index & $28.7 \pm 5.3$ & $29.2 \pm 5.0$ & $28.3 \pm 5.7$ \\
\hline Mean gestational age at enrollment — wk & $27.3 \pm 3.8$ & $27.6 \pm 3.9$ & $26.9 \pm 3.7$ \\
\hline \multicolumn{4}{|l|}{ Gravidity } \\
\hline Median & 2.0 & 2.0 & 2.0 \\
\hline Interquartile range & $2.0-3.0$ & $2.0-3.0$ & $2.0-3.0$ \\
\hline \multicolumn{4}{|l|}{ Parity } \\
\hline Median & 1.0 & 1.0 & 1.0 \\
\hline Interquartile range & $1.0-2.0$ & $1.0-2.0$ & $1.0-2.0$ \\
\hline \multicolumn{4}{|l|}{ HAART - no. (\%) } \\
\hline At enrollment & $60(30.9)$ & $22(22.0) \dagger$ & $38(40.4) \dagger$ \\
\hline At delivery & $77(39.7)$ & $33(33.0)$ & $44(46.8)$ \\
\hline \multicolumn{4}{|l|}{$\begin{array}{l}\text { ART for prevention of mother-to-child HIV transmission } \\
\qquad- \text { no. (\%) }\end{array}$} \\
\hline At enrollment & $86(44.3)$ & $54(54.0) \dagger$ & $32(34.0) \uparrow$ \\
\hline At delivery & $87(44.9)$ & $52(52.0) \mathbb{\int}$ & $35(37.2) \mathbb{\int}$ \\
\hline \multicolumn{4}{|l|}{ CD4+ T-lymphocyte count - cells $/ \mathrm{mm}^{3}$} \\
\hline \multicolumn{4}{|l|}{ At enrollment $q$} \\
\hline Median & 393.5 & 410.0 & 379.0 \\
\hline Interquartile range & $271.0-557.0$ & $284.0-572.0$ & $245.0-550.0$ \\
\hline \multicolumn{4}{|l|}{ At post-vaccination visit $\|$} \\
\hline Median & 399.5 & 371.0 & 409.0 \\
\hline Interquartile range & $265.0-499.0$ & $255.0-499.0$ & $274.0-500.0$ \\
\hline \multicolumn{4}{|l|}{ At delivery } \\
\hline Median & 446.5 & 441.0 & 508.0 \\
\hline Interquartile range & $330.0-599.00$ & $303.0-549.0$ & $346.0-673.0$ \\
\hline \multicolumn{4}{|l|}{ HIV-I viral load - copies/ml } \\
\hline \multicolumn{4}{|l|}{ At enrollment ${ }^{\dagger}{ }^{\prime} \dagger$} \\
\hline Median & 1067.0 & 1679.0 & 399.0 \\
\hline Interquartile range & $61.0-13,923.0$ & $118.5-15,906.5$ & $39.0-9990.0$ \\
\hline \multicolumn{4}{|l|}{ At post-vaccination visittit } \\
\hline Median & 620.0 & $812.0 \sqrt{5}$ & $292.5 \int$ \\
\hline Interquartile range & $39.0-8278.0$ & $130.0-13,221.0$ & $39.0-3056.0$ \\
\hline \multicolumn{4}{|l|}{ At delivery $\int \mathbb{\int}$} \\
\hline Median & 265.0 & 222.0 & 356.0 \\
\hline Interquartile range & $54.0-2040.0$ & $64.0-2262.0$ & $39.0-1880.0$ \\
\hline \multicolumn{4}{|l|}{ Fetuses and newborns } \\
\hline Total no. of live-born infants & 188 & 100 & 88 \\
\hline Twin birth - no. (\%) & $10(5.3)$ & $6(6.0)$ & $4(4.5)$ \\
\hline \multicolumn{4}{|l|}{ Type of delivery - no. (\%) } \\
\hline Normal vaginal & $111(59.0)$ & $58(58.0)$ & $53(60.2)$ \\
\hline Cesarian section & $77(41.0)$ & $42(42.0)$ & $35(39.8)$ \\
\hline Preterm birth, gestational age $<37$ wk — no. (\%) & $26(13.8)$ & $13(13.0)$ & $13(14.8)$ \\
\hline
\end{tabular}




\begin{tabular}{|c|c|c|c|}
\hline Characteristic or Outcome & Overall & IIV3 & Placebo \\
\hline \multicolumn{4}{|l|}{ Overall birth weight — kgף } \\
\hline Median & 3.0 & 2.9 & 3.0 \\
\hline Range & $0.8-4.3$ & $0.8-4.3$ & $1.9-4.0$ \\
\hline \multicolumn{4}{|c|}{ Birth weight of infants born during influenza season $-\mathrm{kg}\|\|$} \\
\hline Median & 3.0 & 3.0 & 3.0 \\
\hline Range & $0.8-4.3$ & $0.8-4.3$ & $1.9-4.1$ \\
\hline Birth weight of $<2500 \mathrm{~g}-$ no. (\%) $₫ 9$ & $29(15.6)$ & $14(14.1)$ & $15(17.2)$ \\
\hline Death after delivery — no. $(\%)^{* * * * *}$ & $4(2.1)$ & $2(2.0)$ & $2(2.3)$ \\
\hline Admission to neonatal nursery — no. (\%) & $9(4.8)$ & $6(6.0)$ & $3(3.4)$ \\
\hline $\begin{array}{l}\text { Plus-minus values are means } \pm S D \text {. AR } \\
\text { P<0.01. } \\
\text { Data are based on } 81 \text { observations in } t \\
\text { P<0.05. } \\
\text { Numbers are based on } 97 \text { observations } \\
\text { Numbers are based on } 78 \text { observations } \\
\text { Numbers are based on } 77 \text { observations } \\
\text { Numbers are based on } 96 \text { observations } \\
\text { Numbers are based on } 77 \text { observations } \\
\text { Numbers are based on } 72 \text { observations } \\
\text { Numbers and percentages are based o } \\
\text { Numbers are based on } 88 \text { observations } \\
\text { Further details on newborn deaths are }\end{array}$ & $\begin{array}{l}\text { HAART high } \\
\text { he placebo } \\
\text { s in the pla } \\
\mathrm{s} \text { in the pla } \\
\mathrm{s} \text { in the pla } \\
\mathrm{s} \text { in the pla } \\
\mathrm{s} \text { in the pla } \\
\mathrm{s} \text { in the pla } \\
\text { d } 87 \text { obser } \\
\mathrm{s} \text { in the pla } \\
\text { entary Appe }\end{array}$ & e placebo & \\
\hline
\end{tabular}

sis stratified according to sex and an analysis limited to births that occurred during the influenza season.

\section{DISCUSSION}

The trials of IIV3 efficacy in HIV-infected and HIV-uninfected pregnant women showed a reduction in confirmed cases of influenza in both cohorts. Furthermore, we observed protection against confirmed influenza among infants of IIV3 recipients who were not exposed to HIV, with a similar trend observed in infants exposed to HIV. IIV3 was found to be safe, and the vaccine was immunogenic in HIV-uninfected women and HIVinfected women, supporting the current WHO recommendations for influenza vaccination during pregnancy. ${ }^{19}$

The vaccine efficacy against confirmed influenza in infants who were not exposed to HIV (45.6\%; 95\% CI, 2.4 to 69.7 ) was similar to that reported in Bangladesh (63\%; 95\% CI, 5 to 85). ${ }^{16}$ However, direct comparisons with the results of the Bangladesh study are limited because of differences in the assays used for viral detection. In addition, influenza virus circulation in Bangla- desh is prolonged and perennial, limiting the generalizability of the Bangladesh study to temperate regions with more discrete influenza seasons, such as South Africa. ${ }^{16}$ The effectiveness of IIV3 vaccination in protecting the infants of pregnant women not infected with HIV is further corroborated by case-control studies in the United States among American Indians (in which most cases were diagnosed through serologic testing) and by a study of influenza-associated hospitalization in a large urban hospital (in which the diagnosis was confirmed through fluorescent antibody testing). ${ }^{20,22}$

It has been proposed that higher HAI titers may be required to provide protection against influenza in children; in adults, an HAI titer of 1:40 or higher is associated with $50 \%$ protection..$^{31,32,33}$ Nevertheless, in our study the proportion of HIV-unexposed newborns in the IIV3 group with HAI titers of $1: 40$ or higher (60 to $82 \%$ ) suggests that this threshold may be associated with approximately $50 \%$ protection against confirmed influenza in such infants. Further studies and analyses are warranted to establish a serocorrelate for the protection of HAI antibodies acquired through the placenta. Another way in which the 
vaccination of pregnant women may protect their infants against influenza involves prevention of the transmission of the virus to the infant by reducing the mother's risk of influenza illness. This is corroborated in part by our study, in which concurrent confirmed influenza infection by homotypic strains occurred more frequently in HIVunexposed infants of placebo recipients than in HIV-unexposed infants of IIV3 recipients; infections with homotypic strains also occurred concurrently in $54.5 \%$ of the mothers of HIV-exposed infants with confirmed influenza illness.

Unlike the study in Bangladesh, in which rates of clinical febrile respiratory illness were reduced by $29 \%$ and $36 \%$ in infants and mothers, respectively ${ }^{16}$ our study did not show any reduction in the less specific outcomes of clinical influenzalike illness or respiratory illness in either infants or mothers. The use of pneumococcal polysaccharide vaccine as the comparator for the control group in the Bangladeshi study, as well as the use of different case definitions, may have contributed to the discrepant findings in the two studies. Observational studies in the United States have also shown that maternal influenza vaccination was not effective in protecting infants against allcause respiratory illness, indicating the lack of specificity of such end points for the evaluation of influenza vaccine efficacy in infants..$^{21,23}$

The vaccine efficacy against confirmed influenza among HIV-uninfected women in our study (per-protocol analysis, 54.4\%; 95\% CI, 19.5 to 74.2) was similar to that reported in an observational study of vaccination against monovalent A/(H1N1)pdm09 in pregnant Norwegian women (70\%; $95 \%$ CI, 66 to 75$)^{33}$ and in a more recent case-control study in the United States (44 to $53 \%) .{ }^{17}$ The vaccine efficacy against confirmed influenza in HIV-infected pregnant women in our study (per-protocol analysis, 70.6\%) was similar to that reported in a trial of IIV3 in South African HIV-infected men and women during the 2008 influenza epidemic (75.5\%). ${ }^{34}$ The efficacy of the vaccine in our study was also similar to that reported in a meta-analysis of HIV-infected adults in high-income countries (66\%; 95\% CI, 36 to 82 ). ${ }^{35}$ Notably, the attack rate among HIV-infected placebo recipients in our study (17.0\%) was greater than that observed among HIV-uninfected placebo recipients $(3.6 \%)$, a finding that highlights the greater susceptibility of HIV-infected persons to influenza, even when the HIV infection is managed with antiretroviral treatment.

Extrapolation of the potential effect of influenza vaccination in pregnancy solely on the basis of HAI immune responses may be affected by pregnancy-inducing immune tolerance, which could influence vaccine effectiveness. ${ }^{4,536}$ The availability of immunogenicity and efficacy data in our nested immunogenicity study helped to address this question. The proportions of HIV-uninfected IIV3 recipients with seroprotective HAI titers for $\mathrm{A} /(\mathrm{H} 1 \mathrm{~N} 1) \mathrm{pdm} 09, \mathrm{~A} / \mathrm{H} 3 \mathrm{~N} 2$, and $\mathrm{B} /$ Victoria were $93.3 \%, 78.0 \%$, and $96.0 \%$, respectively, with corresponding overall vaccine efficacy against confirmed influenza of $54.4 \%$. These findings suggest that the threshold of 1:40 or higher was predictive of an anticipated vaccine efficacy of $50 \%$, as proposed for healthy adults. ${ }^{31}$ Theoretically, the rate of naturally acquired immunity among placebo recipients (20 to $45 \%$ ) could have decreased the true efficacy of the vaccine, a consideration that would not be required in an immunologically naive placebo group of women not infected with influenza.

Among IIV3 recipients in the HIV-infected cohort, HAI titers of 1:40 or higher were observed for $\mathrm{A} / \mathrm{H} 3 \mathrm{~N} 2$ and $\mathrm{A} /(\mathrm{H} 1 \mathrm{~N} 1) \mathrm{pdm} 09$ (48.6\% and $68.6 \%$, respectively). Consequently, the HAI titer of 1:40 or higher, as a relative serocorrelate of protection, would have paradoxically underestimated vaccine efficacy against confirmed influenza among the HIV-infected women (reported as $70.6 \%$ ). This observation is consistent with findings from our previous study involving HIV-infected adults. ${ }^{34}$ Among the HIV-infected cohort, IIV3 did not affect the plasma HIV-1 viral load, minimizing concern about the possibility of an increased risk of vertical transmission of HIV in vaccinees. ${ }^{37,38}$

One of the limitations of our study is the fact that it was conducted at a single center; the generalizability of the findings needs to be corroborated. Furthermore, the timing of enrollment was contingent on the commercial availability of IIV3. Although we initiated enrollment within 1 week after vaccine availability, vaccination extended into the influenza season, with some births occurring after the influenza season. The difference in duration of exposure to the influenza virus between the women and their infants limited our ability to make a direct comparison of the attack 


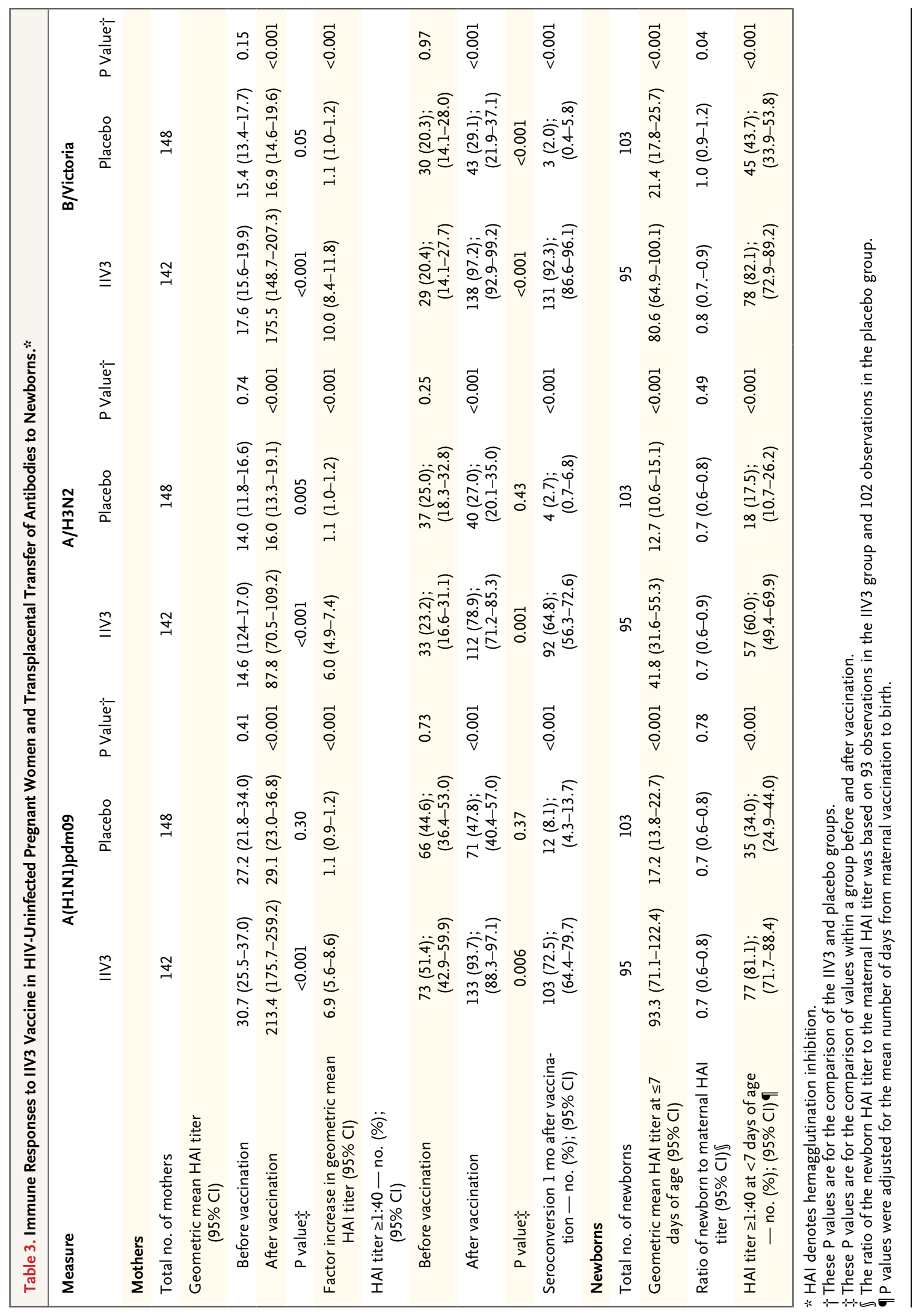




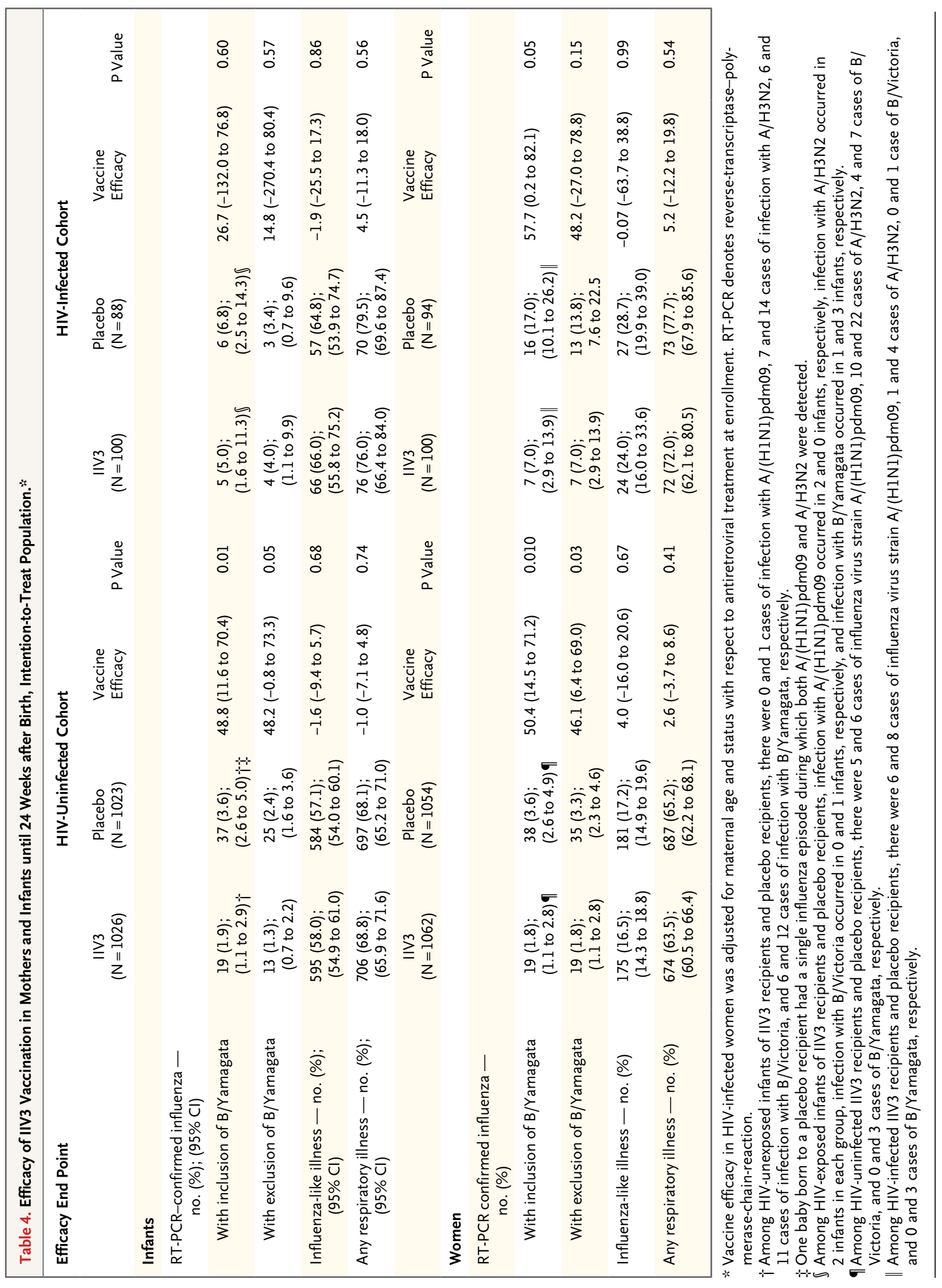




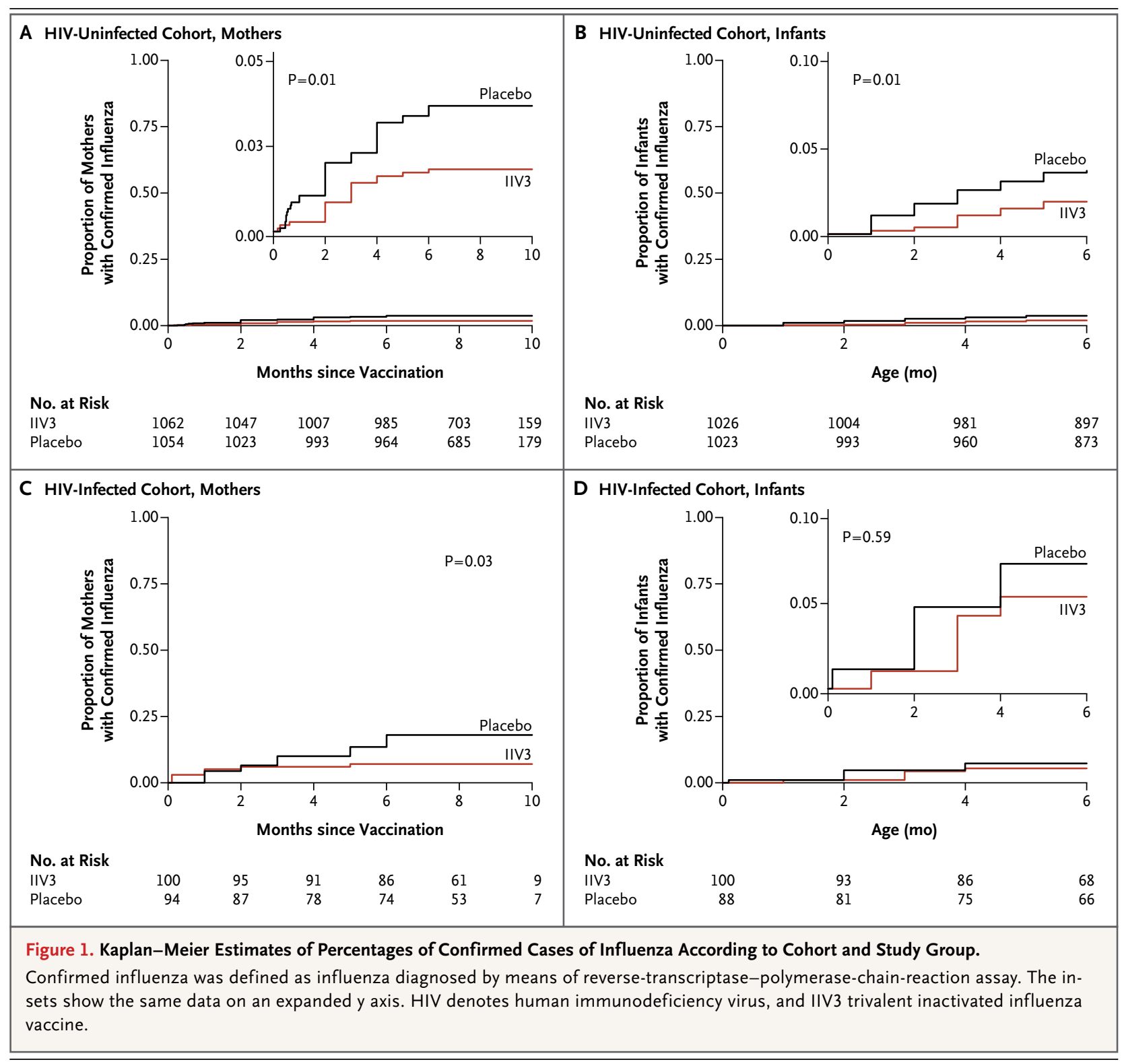

rates between them and between these infants and those in the Bangladeshi study, for whom exposure was probably more prolonged. ${ }^{16,39} \mathrm{An}$ other limitation of our study is the fact that the evaluation of vaccine efficacy among HIV-infected women was a secondary objective; nevertheless, its efficacy in these women was indicated by the high attack rate in the placebo group. However, the study lacked power to evaluate vaccine efficacy among the infants of HIV-infected women, for whom the point estimate was similar to that for HIV-uninfected infants in the per- protocol analysis. Although 23\% of the HIV-infected and uninfected immunogenicity cohorts were outside the protocol-defined windows for obtaining post-vaccination blood samples, the results were similar to those included in the exploratory immunogenicity analysis with an expanded window. An inadvertent occurrence in the study was the difference in HIV disease characteristics between the placebo group and the IIV3 group, including the fact that placebo recipients were more likely to be receiving highly active antiretroviral therapy. However, this factor would 
have biased the results in the direction of a reduced risk of influenza in the placebo group. ${ }^{7}$ In addition, in HIV-infected women, the lower HAI titers among placebo recipients as compared with IIV3 recipients could have been due to differences in HIV disease stage between the groups.

In conclusion, these data show that IIV3 vaccination in pregnant HIV-uninfected and HIVinfected African women was immunogenic and provided protection against confirmed influenza. The vaccination was also effective in HIV-unexposed infants up until 24 weeks after birth.

The contents of this report are solely the responsibility of the authors and do not necessarily represent the official views of their institutions or organizations or of the study sponsors.

Supported by grants from the Bill and Melinda Gates Foundation (OPP1002747), the National Institutes of Health, National Center for Advancing Translational Sciences Colorado Clinical and Translational Sciences Institute (UL1 TR000154, for REDCap), the South African Research Chairs Initiative of the Department of Science and Technology and National Research Foundation in Vaccine-Preventable Diseases, and the Respiratory and Meningeal Pathogens Research Unit of the Medical Research Council.

Dr. Madhi reports receiving lecture fees and fees for serving on advisory boards from GlaxoSmithKline, Pfizer, and Sanofi Pasteur and grant support from Novartis, GlaxoSmithKline, Pfizer, Sanofi Pasteur, and MedImmune; and Dr. Weinberg consulting fees from Merck and grant support from Merck, MedImmune, Becton Dickinson, Roche, and GlaxoSmithKline; and Dr. Violari fees for serving on a data and safety monitoring board from Janssen and grant support from Janssen, Aeras, Gilead, and the Drugs for Neglected Diseases Initiative. No other potential conflict of interest relevant to this article was reported.

Disclosure forms provided by the authors are available with the full text of this article at NEJM.org.

We thank all the study participants; the staff of the Departments of Obstetrics, Neonatology, and Paediatrics at Chris Hani-Baragwanath Academic Hospital, Soweto, South Africa, for their dedication to their patients, including our trial participants; the study midwives, nurses, laboratory staff, counselors, and data capturers; and Niteen Wairagkar, program officer acting on behalf of the Bill and Melinda Gates Foundation.

\section{APPENDIX}

From the Medical Research Council, Respiratory and Meningeal Pathogens Research Unit (S.A.M., C.L.C., L.K., A.H., S.J., P.V.A., N.N., K.P.K., M.C.N.), the Department of Science and Technology-National Research Foundation, Vaccine-Preventable Diseases (S.A.M., C.L.C., L.K., A.H., S.J., P.V.A., N.N., M.C.N.), and the Perinatal HIV Research Unit (A.V.), University of the Witwatersrand, the National Institute for Communicable Diseases, the National Health Laboratory Service, Centre for Vaccines and Immunology (S.A.M., F.T., M.V.), Johannesburg, and the Department of Medical Virology, University of Pretoria, Pretoria (M.V.) — all in South Africa; the School of Medicine and Children's Hospital, University of Colorado (A.W.), the Department of Pediatrics, Medicine and Pathology, University of Colorado School of Medicine (E.A.F.S.), and the Center for Global Health, Department of Epidemiology, Colorado School of Public Health (E.A.F.S.) - all in Aurora, Colorado; the Department of Medicine and Department of Global Health, University of Washington (J.R.O.), and the Vaccine Access and Delivery Global Program, PATH (J.R.O., K.M.N.) - both in Seattle; and the Hubert Department of Global Health, Rollins School of Public Health, and the Division of Infectious Diseases, School of Medicine, Emory University, Atlanta (K.P.K.)

\section{REFERENCES}

1. Meeting of the Strategic Advisory Group of Experts on Immunization, April 2012 - conclusions and recommendations. Wkly Epidemiol Rec 2012;87:20116.

2. Neuzil KM, Reed GW, Mitchel EF, Simonsen L, Griffin MR. Impact of influenza on acute cardiopulmonary hospitalizations in pregnant women. Am J Epidemiol 1998;148:1094-102.

3. Louie JK, Acosta M, Jamieson DJ, et al. Severe 2009 H1N1 influenza in pregnant and postpartum women in California. N Engl J Med 2010;362:27-35.

4. Jamieson DJ, Theiler RN, Rasmussen SA. Emerging infections and pregnancy. Emerg Infect Dis 2006;12:1638-43.

5. Schlaudecker EP, McNeal MM, Dodd CN, Ranz JB, Steinhoff MC. Pregnancy modifies the antibody response to trivalent influenza immunization. J Infect Dis 2012;206:1670-3.

6. Cohen C, Simonsen L, Sample J, et al. Influenza-related mortality among adults aged 25-54 years with AIDS in South Africa and the United States of America. Clin Infect Dis 2012;55:996-1003.
7. Neuzil KM, Coffey CS, Mitchel EF Jr, Griffin MR. Cardiopulmonary hospitalizations during influenza season in adults and adolescents with advanced HIV infection. J Acquir Immune Defic Syndr 2003; 34:304-7.

8. Archer B, Cohen C, Naidoo D, et al. Interim report on pandemic $\mathrm{H} 1 \mathrm{~N} 1$ influenza virus infections in South Africa, April to October 2009: epidemiology and factors associated with fatal cases. Euro Surveill 2009;14:19369.

9. Staprans SI, Hamilton BL, Follansbee SE, et al. Activation of virus replication after vaccination of HIV-1-infected individuals. J Exp Med 1995;182:1727-37. 10. Nelson KE, Clements ML, Miotti P, Cohn S, Polk BF. The influence of human immunodeficiency virus (HIV) infection on antibody responses to influenza vaccines. Ann Intern Med 1988;109:383-8.

11. Miotti PG, Nelson KE, Dallabetta GA Farzadegan H, Margolick J, Clements ML. The influence of HIV infection on antibody responses to a two-dose regimen of influenza vaccine. JAMA 1989;262:77983.
12. Fuller JD, Craven DE, Steger KA, Cox $\mathrm{N}$, Heeren TC, Chernoff D. Influenza vaccination of human immunodeficiency virus (HIV)-infected adults: impact on plasma levels of HIV type 1 RNA and determinants of antibody response. Clin Infect Dis 1999;28:541-7.

13. Benne CA, Kroon FP, Harmsen M, Tavares L, Kraaijeveld CA, De Jong JC. Comparison of neutralizing and hemagglutination-inhibiting antibody responses to influenza A virus vaccination of human immunodeficiency virus-infected individuals. Clin Diagn Lab Immunol 1998;5: 114-7.

14. Kroon FP, van Dissel JT, de Jong JC, Zwinderman K, van Furth R. Antibody response after influenza vaccination in HIV-infected individuals: a consecutive 3-year study. Vaccine 2000;18:3040-9. 15. Hulka JF. Effectiveness of polyvalent influenza vaccine in pregnancy: report of a controlled study during an outbreak of Asian influenza. Obstet Gynecol 1964;23: 830-7.

16. Zaman K, Roy E, Arifeen SE, et al. Effectiveness of maternal influenza immu- 
nization in mothers and infants. $\mathrm{N}$ Engl $\mathrm{J}$ Med 2008;359:1555-64.

17. Thompson MG, Li DK, Shifflett P, et al. Effectiveness of seasonal trivalent influenza vaccine for preventing influenza virus illness among pregnant women: a population-based case-control study during the 2010-2011 and 2011-2012 influenza seasons. Clin Infect Dis 2014;58: 449-57.

18. Ortiz JR, Englund JA, Neuzil KM. Influenza vaccine for pregnant women in resource-constrained countries: a review of the evidence to inform policy decisions. Vaccine 2011;29:4439-52.

19. Vaccines against influenza WHO position paper - November 2012. Wkly Epidemiol Rec 2012;87:461-76.

20. Benowitz I, Esposito DB, Gracey KD, Shapiro ED, Vázquez M. Influenza vaccine given to pregnant women reduces hospitalization due to influenza in their infants. Clin Infect Dis 2010;51:1355-61.

21. Black SB, Shinefield HR, France EK, et al. Effectiveness of influenza vaccine during pregnancy in preventing hospitalizations and outpatient visits for respiratory illness in pregnant women and their infants. Am J Perinatol 2004;21:333-9.

22. Eick AA, Uyeki TM, Klimov A, et al. Maternal influenza vaccination and effect on influenza virus infection in young infants. Arch Pediatr Adolesc Med 2011;165: 104-11.

23. France EK, Smith-Ray R, McClure D, et al. Impact of maternal influenza vaccination during pregnancy on the incidence of acute respiratory illness visits among infants. Arch Pediatr Adolesc Med 2006; 160:1277-83.

24. Radin JM, Katz MA, Tempia S, et al. Influenza surveillance in 15 countries in
Africa, 2006-2010. J Infect Dis 2012;206: Suppl 1:S14-S21.

25. Poehling KA, Edwards KM, Weinberg $\mathrm{GA}$, et al. The underrecognized burden of influenza in young children. $\mathrm{N}$ Engl J Med 2006;355:31-40.

26. Englund JA, Walter E, Black $S$, et al. Safety and immunogenicity of trivalent inactivated influenza vaccine in infants: a randomized double-blind placebo-controlled study. Pediatr Infect Dis J 2010;29: 105-10.

27. Recommended viruses for influenza vaccines for use in the 2011 southern hemisphere influenza season. Geneva: World Health Organization, 2010 (http://www .who.int/influenza/vaccines/virus/ recommendations/201009_Recommendation.pdf?ua=1)

28. VAXIGRIP. Inactivated influenza vaccine (Split Virion) product information. France: Sanofi Pasteur, 2011 (http://www .sanofipasteur.com/en).

29. Table for Grading the Severity of Adult and Pediatric Adverse Events Version 1.0 - December 2004 (clarification dated August 2009) (http://rsc.tech-res .com/safetyandpharmacovigilance).

30. Harris PA, Taylor R, Thielke R, Payne J, Gonzalez N, Conde JG. Research electronic data capture (REDCap) - a metadata-driven methodology and workflow process for providing translational research informatics support. J Biomed Inform 2009;42:377-81.

31. Hobson D, Curry RL, Beare AS, WardGardner A. The role of serum haemagglutination-inhibiting antibody in protection against challenge infection with influenza A2 and B viruses. J Hyg (Lond) 1972;70: 767-77.

32. Black S, Nicolay U, Vesikari T, et al.
Hemagglutination inhibition antibody titers as a correlate of protection for inactivated influenza vaccines in children. Pediatr Infect Dis J 2011;30:1081-5.

33. Håberg SE, Trogstad L, Gunnes N, et al. Risk of fetal death after pandemic influenza virus infection or vaccination. N Engl J Med 2013;368:333-40.

34. Madhi SA, Maskew M, Koen A, et al. Trivalent inactivated influenza vaccine in African adults infected with human immunodeficient virus: double blind, randomized clinical trial of efficacy, immunogenicity, and safety. Clin Infect Dis 2011;52:128-37.

35. Anema A, Mills E, Montaner J, Brownstein JS, Cooper C. Efficacy of influenza vaccination in HIV-positive patients: a systematic review and meta-analysis. HIV Med 2008;9:57-61.

36. Robinson DP, Klein SL. Pregnancy and pregnancy-associated hormones alter immune responses and disease pathogenesis. Horm Behav 2012;62:263-71.

37. Jamieson DJ, Sibailly TS, Sadek R, et al. HIV-1 viral load and other risk factors for mother-to-child transmission of HIV1 in a breast-feeding population in Cote d'Ivoire. J Acquir Immune Defic Syndr 2003;34:430-6.

38. Tasker SA, O'Brien WA, Treanor JJ, et al. Effects of influenza vaccination in HIV-infected adults: a double-blind, placebo-controlled trial. Vaccine 1998;16: 1039-42.

39. Influenza surveillance report South Africa. National Institute for Communicable Diseases: a division of National Health Laboratory Service, May 2014 (http://www.nicd.ac.za/?page=seasonal_ influenza\&id $=72$ ).

Copyright $\odot 2014$ Massachusetts Medical Society. 